\title{
IdeAs
}

Idées d'Amériques

$17 \mid 2021$

Villes et culture dans les Amériques

\section{Hidroituango: Un desastre socioambiental con responsabilidad internacional}

Isabel Cristina Zuleta

\section{(2) OpenEdition}

\section{Journals}

Edición electrónica

URL: https://journals.openedition.org/ideas/10005

DOI: 10.4000/ideas.10005

ISSN: 1950-5701

Editor

Institut des Amériques

Referencia electrónica

Isabel Cristina Zuleta, «Hidroituango: Un desastre socioambiental con responsabilidad internacional», IdeAs [En línea], 17 | 2021, Publicado el 01 marzo 2021, consultado el 03 junio 2021. URL: http:// journals.openedition.org/ideas/10005 ; DOI: https://doi.org/10.4000/ideas. 10005

Este documento fue generado automáticamente el 3 junio 2021.

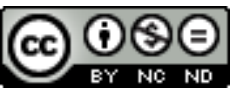

IdeAs - Idées d'Amériques est mis à disposition selon les termes de la licence Creative Commons Attribution - Pas d'Utilisation Commerciale - Pas de Modification 4.0 International. 


\title{
Hidroituango: Un desastre socioambiental con responsabilidad internacional
}

\author{
Isabel Cristina Zuleta
}

\section{NOTA DEL EDITOR}

Con el apoyo de las coordinadoras del dossier "Proteger a los líderes sociales en el Postacuerdo de paz", Laetitia Braconnier-Moreno y Laura Cahier.

1 El proyecto hidroeléctrico "Hidroituango" está ubicado en el Cañón del Río Cauca en el departamento de Antioquia, Colombia. Afecta a un profundo cañón conformado por dos cordilleras, la central y la occidental, formaciones de montañas jóvenes e inestables. En estas se albergaban especies endémicas cuyo hábitat era el Bosque Seco Tropical, ecosistema en vía de extinción en el planeta (ver imagen 1). Desde el inicio de las obras en 2009, el proyecto Hidroituango ha dejado miles de familias de varios municipios en condiciones de miseria, de inseguridad alimentaria, sin vivienda ni medios de subsistencia digna, atravesadas por la angustia permanente ${ }^{1}$. 


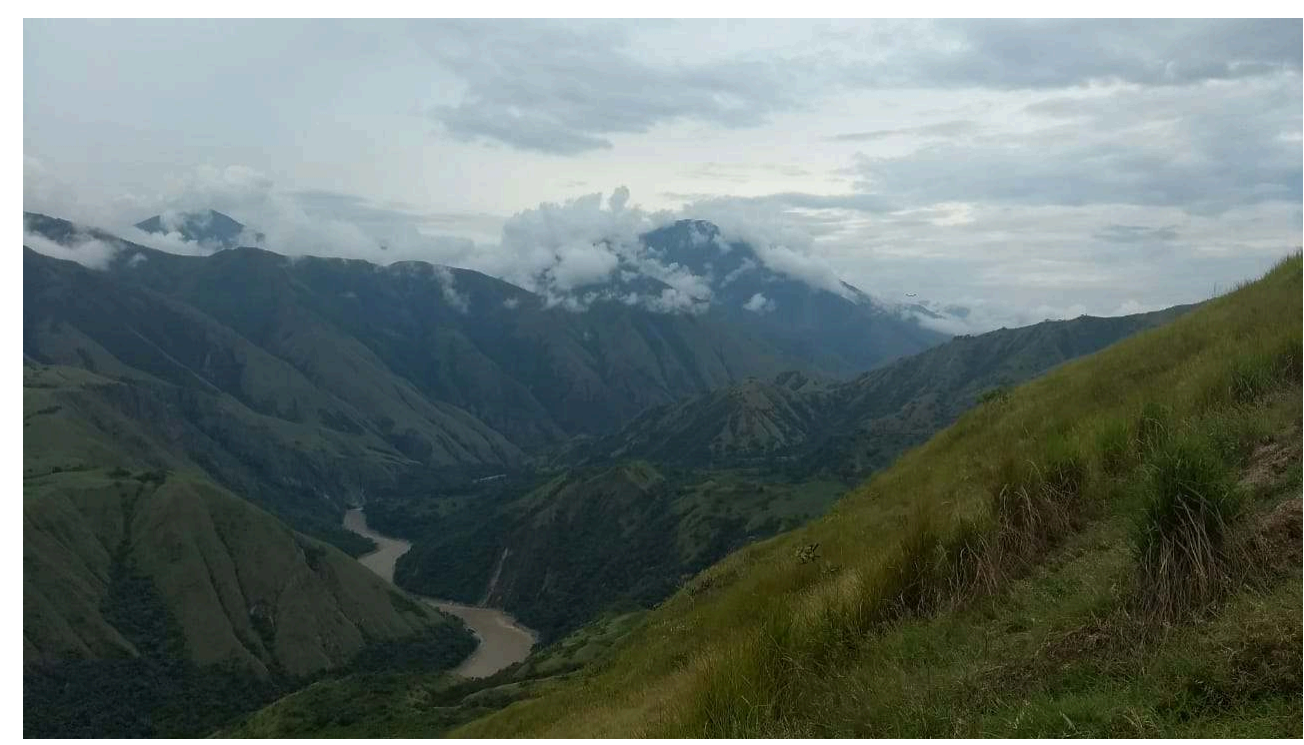

Imagen 1: Cañon del Río Cauca, Antioquia, Colombia.

Fuente: Movimiento Ríos Vivo

\section{El desastre sociocultural y ambiental anunciado}

2 Es necesario volver a los hechos para entender los antecedentes de la crisis multidimensional provocada por el megaproyecto de represa hidroeléctrica Hidroituango. En abril de 2018, la entidad Empresas Públicas de Medellín (EPM) en conjunto con la Gobernación de Antioquia, máxima autoridad estatal en el orden departamental, responsables del proyecto, iniciaron la inundación del embalse, mediante la obstrucción de un gigante río - el río Cauca. Ocurrió cuando aún no habían terminado las obras necesarias para una operación de tal magnitud. Cabe describirla: estaba conformada por un muro de 225 metros de alto, una casa de máquinas para ocho turbinas (para generar $2.400 \mathrm{MW}$ de energía) con un gigantesco entramado de túneles al interior de la montaña, cientos de puentes, zonas de depósitos y kilómetros de vías nuevas, una extensión de 79 kilómetros de inundación, más de 30 hectáreas afectadas, campamentos con la capacidad de albergar a cinco mil obreros, tres enormes líneas de transmisión de alto voltaje, subestaciones, cementeras y todo tipo de obras asociadas. Estas obras se realizaron sin que exista certeza sobre la estabilidad de la zona, pese a que en el área de construcción hay más de siete fallas geológicas en su mayoría activas.

3 En efecto, EPM adelantó obras y realizó intervenciones sin permiso de la Autoridad Nacional de Licencias Ambientales (ANLA). Esta le ordenó contratar un peritaje que recayó en la consultora internacional Pöyry. Las comunidades desconfiaron de este contrato por múltiples motivos, entre los cuales se encuentra el hecho que peritaje esté pagado por la misma entidad constructora de Hidroituango, es decir, Empresas Públicas de Medellín; también porque Pöyry presta servicios de ingeniería a proyectos de energía, minería e infraestructura en proyectos en Latinoamérica, tan controvertidos que la obligaron a cambiar su nombre; en fin, porque el contrato entre EPM y esta consultora se haya modificado en cinco ocasiones sin aún haber presentado resultados.

4 Frente a estas irregularidades, el Movimiento Ríos Vivos, acompañado por un colectivo de abogados, había realizado previamente a la inundación una demanda de nulidad de la licencia ambiental otorgada a Hidroituango por la ANLA. Así, al peritaje ordenado 
por la autoridad ambiental, se le sumó la orden de un Juez de control de garantías, el juez 75 penal de Bogotá, quien ordenó el 18 de junio de 2019 la realización de un estudio independiente para determinar la estabilidad o no del macizo rocoso y la viabilidad o no del proyecto Hidroituango, mediante una mesa técnica que integrará a las víctimas del proyecto. El 11 de diciembre de 2020, el mismo juez confirmó las medidas cautelares para proteger a los pobladores, estableciendo los numerosos incumplimientos del Estado colombiano hasta el momento.

5 Pese a las incertidumbres y los riesgos, los constructores inundaron y destruyeron miles de hectáreas de tierra, sin dar aviso a las familias que han habitado ancestralmente dicho territorio. A causa de faltas graves de debida diligencia ${ }^{2}$ en una época de torrenciales lluvias, la operación fracasó: EPM tomó la decisión de empezar el llenado taponando con cemento los túneles, sin haber terminado el muro de la represa, el vertedero, ni el túnel de descarga intermedia que era el encargado del caudal ecológico. Muchas de las familias quedaron atrapadas por las aguas, debido a los tapones de cemento que obstaculizaron su paso por los dos túneles de desviación, mientras un tercer túnel no soportó la presión y colapsó. La población aguas abajo del muro fue evacuada y aguas arriba rescatada, pero en ambos casos abandonada a su suerte, con escasas intervenciones por parte de la institucionalidad.

6 La zona quedó altamente inestable por el súbito llenado. El 16 de mayo de 2018, el túnel que había colapsado se destapó por escasos minutos y produjo una avalancha que destruyó viviendas e infraestructuras comunitarias como puentes, hospitales, escuelas, iglesias, cementerios con enterramientos de víctimas del conflicto. Los puentes bloqueados generaron la desconexión entre ambos lados del río Cauca. Se quebró la economía: debajo del agua quedaron los medios de vida y subsistencia de las comunidades, perdieron sus viviendas y trabajo pescadores y barequeros. Quedaron en una situación de supervivencia diaria, del rebusque de los alimentos de cualquier forma. Las miles de personas humildes habitando este territorio percibieron el bloqueo de este gran río como un acto de desprecio por su humanidad. Desde entonces su vida ha estado en vilo.

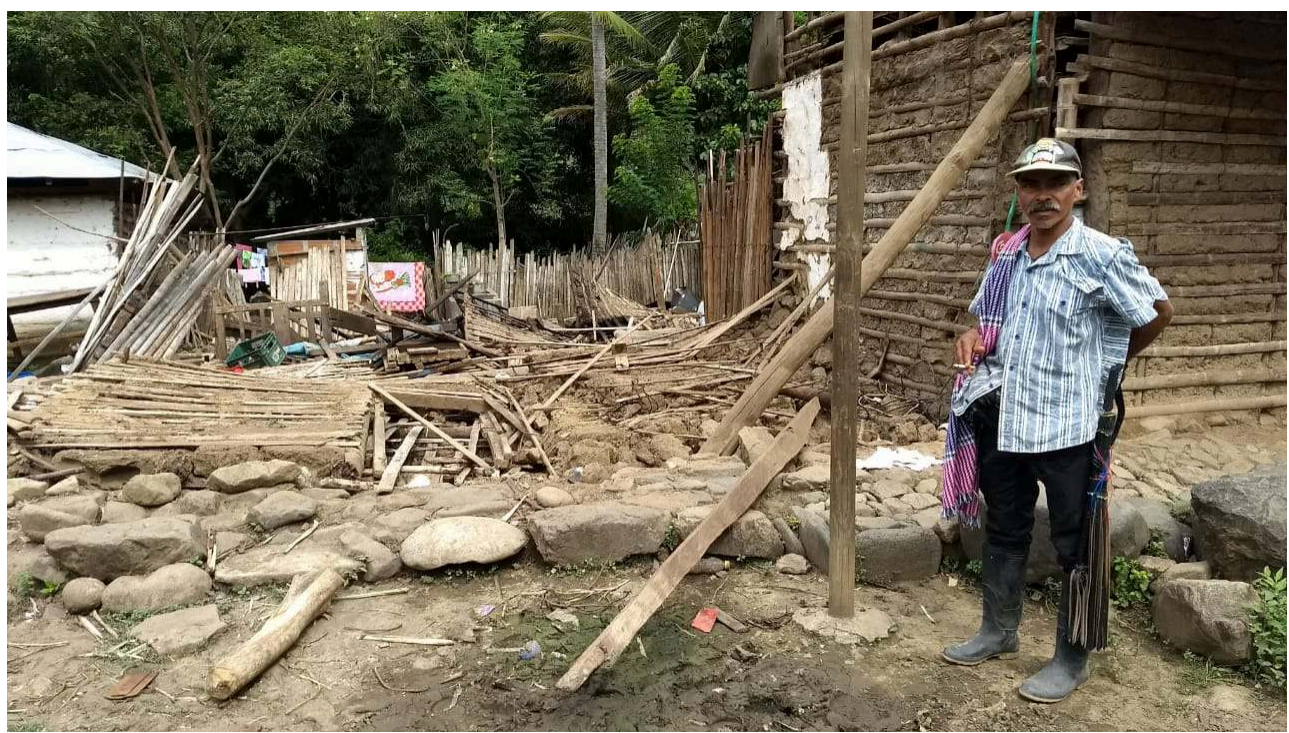

Imagen 2: Municipio afectado después de la inundación de Hidroituango en 2018.

Fuente: Movimiento Ríos Vivos 


\section{Revictimización y resistencia comunitaria}

7 Luego de estos daños a las comunidades y al medio ambiente, no se garantizó reparación para las personas desplazadas por el proyecto, quienes ya se encontraban en una situación de vulnerabilidad. En efecto, y como hecho agravante, dicho proyecto hidroeléctrico se construye en una zona en donde la población ha sufrido el rigor de la guerra y la disputa territorial entre distintos grupos ilegales. Por lo menos el $60 \%$ de los habitantes de la zona afectada por la represa fueron antes víctimas del conflicto armado. 2904 personas han sido desaparecidas desde los años 80's, lo que ha llevado a la Jurisdicción Especial para la Paz, competente desde el año 2017 para juzgar a algunos responsables del conflicto armado colombiano, a tomar medidas cautelares para adelantar investigaciones por los derechos de las víctimas.

8 La represa y el desplazamiento forzado que engendra vulneran la memoria colectiva local sobre el conflicto. Como consecuencia de la inundación, fosas comunes y lugares de inhumación en donde yacen cuerpos no identificados, han sido amenazadas de destrucción. Dichas huellas materiales de por sí difíciles de hallar, están desapareciendo, vulnerando el derecho a la verdad y justicia de las familias que buscan desde hace décadas a sus seres queridos. Ahora bien, este crimen de crímenes no ha cesado; los líderes siguen siendo asesinados y desaparecidos por grupos paramilitares que despliegan terror sobre la colectividad en su conjunto y no sólo sobre los movimientos sociales. La permanencia de actores criminales evidencia, para las comunidades, que el Estado, por una parte, no tiene el control sobre el territorio, y por otra, que predomina la figura de un Estado corporativo pues la empresa EPM toma decisiones de toda índole, incluso en materia de movilidad y seguridad.

Las comunidades afectadas por dicha violencia sociopolítica ejercida por el Estado se han organizado en 2008 en el Movimiento Ríos Vivos, una asociación de segundo nivel que agrupa a 17 colectivos de pescadores, barequeros - mineros ancestrales y artesanales - campesinos, mujeres y jóvenes. Surge como respuesta a los atropellos por parte de la empresa constructora, y principalmente los desalojos forzosos, sin reubicación de las familias o respeto por los derechos de las comunidades. Los pobladores denuncian especialmente la práctica del abuso de la fuerza a la hora de sacar del territorio a sus habitantes ancestrales para dar paso a una lógica de desarrollo contraria a la cultura cañonera.

En primer lugar, sus solicitudes radican en la instalación de las condiciones de diálogo garantía y protección de derechos -, considerando este como un factor de protección y participación en las decisiones que les afectan en especial cuando se trata de los derechos humanos y ambientales. El Movimiento Ríos Vivos ha insistido a las empresas y bancos que los escuchen. Envió en ese sentido información concreta sobre los impactos y riesgos de este megaproyecto, y de manera especial, ha puntualizado sobre el contexto del conflicto armado y las implicaciones de sus inversiones en una zona sembrada de minas antipersonales, con una historia marcada por disputas territoriales continuas entre actores armados, enfrentamientos, asesinatos, centenares de masacres, miles de desaparecidos. En fin, los líderes exigieron el retiro de las inversiones y la reparación integral de las personas desplazadas. 


\section{Un proyecto legal y público con responsabilidad internacional}

11 Cabe resaltar que el proyecto Hidroituango es un proyecto público, pues está ejecutado por una Empresa pública - las EPM - con la mayoría accionaria de la Gobernación de Antioquia. Oponerse a un proyecto público tiene implicaciones particulares: en ese caso, la comunidad no está en situación de riesgo por oponerse a actividades ilícitas, sino lícitas, y el rol del Estado como mediador, protector y garante de derechos se desdibuja en su conflicto de intereses entre los cuales, privilegia el posible lucro por encima del bienestar de las comunidades. El Sistema Interamericano de Derechos Humanos hace eco de estas denuncias surgidas del territorio, instando al Estado ser garante de los derechos a la vida de estos líderes.

Además, el $64 \%$ del proyecto está financiado con recursos internacionales como el Banco Interamericano de Desarrollo (BID) y bancos privados (ver infografía). Estas inversiones públicas e internacionales, como entes legales, han incidido en el desplazamiento al igual que las actividades ilícitas de los actores armados, especialmente paramilitares. El conjunto de estos actores participa en ese sentido de un mismo ambiente de desprotección y ecosistema de violencia ${ }^{3}$ que obliga a la población a desplazarse o permanecer en el territorio bajo múltiples riesgos. Ríos Vivos considera a estas entidades responsables en el desastre social y ambiental de Hidroituango, en la medida en que estaban informadas de los riesgos al invertir en un proyecto que afecta a comunidades víctimas del conflicto armado vulnerando sus derechos.

\section{Algunas de las empresas y entidades financieras involucradas en Hidroituango.}

Ind. Electromec. GH S.A.; MAPFRE, Banco BBVA y Santander de España; banco KFW, SUEDKABEL GmbH, Helmut Friedrich Miller de Alemania, ALIMAK HEK, Atlas Copco y Scania de Suecia, Consorcios de diferentes nacionalidades como el Consorcio Aqualogus/ Artelia /E\&A Portugues / Frances / Colombiano. SIEMENS TRANSFORMER (GUANZHOU) Co. Ltd. Banco Industrial y Comercial de China (ICBC), Fondo Chino de Cofinanciamiento para América Latina y el Caribe $(\mathrm{CHC})$ de China; ATB RIVA CALZONI S.p.A. de Italia; Klohn Crippen Berger Ltda Klohn Crippen Berger Ltda, Hatch S.A.S. EDC y Caja de Pensiones de Quebec de Canadá; IMPSA de Argentina; Camargo Correa, GE Energías Renováveis Ltda, el banco BNDES de Brasil.BNP Paribas, belga y francés

Imagen 3: Empresas y entidades financieras involucradas en Hidroituango.

Fuente: Movimiento Ríos Vivos

En el año 2018, más de 400 habitantes de la zona presentaron una queja formal ante el Mecanismo independiente de consulta e investigación del BID. No obstante, aunque en 
la actualidad se realiza una investigación con cinco expertos internacionales para determinar el cumplimiento o no del proyecto con las políticas del BID, dicho mecanismo no contempla la posibilidad de solucionar de fondo la problemática y reparar a las comunidades afectadas por los daños causados. En la mayoría de los casos, a pesar de las investigaciones en curso y de la evidencia abundante de sus denuncias, las empresas y la banca se niegan a reconocer los daños que siguen causando el proyecto a la población.

\section{Una disputa sociocultural, ambiental y espiritual}

Este conflicto se sitúa en el plano socio ambiental y cultural: pone a prueba visiones en disputa sobre el desarrollo, pues los defensores del ambiente y de la naturaleza se enfrentan a lo que consideran una cultura mayoritaria de la destrucción. El daño territorial, así como la inundación de los cuerpos de personas desaparecidas, afectan, además, la vida espiritual ${ }^{4}$. En este sentido, los participantes del Movimiento han puesto en su repertorio de acción actividades para el duelo y las búsquedas de personas desaparecidas.

Más allá del litigio jurídico, el Movimiento, a través de encuentros y acciones pedagógicas para repensar el territorio, busca generar una reflexión sobre los daños y las represalias que se siguen viviendo en los territorios. Entre otros ejercicios, sus líderes han llevado a la comunidad a participar en actividades de reforestación autónoma para rescatar procesos ecológicos que se han perdido. Con los pescadores que conocen la dinámica fluvial, han trabajado para recuperar la diversidad de los ríos. Uno de los trasfondos del proceso recae en efecto en el reconocimiento de los saberes ancestrales de las comunidades, que logra en la práctica la recuperación efectiva de los ecosistemas.

\section{Mecanismos autónomos para proteger la vida}

Con base en la constatación que la sociedad mayoritaria no ha ayudado a crear estos espacios de diálogo con las comunidades, espacios que son el primer paso para su respeto y protección, este es otro de los retos que debe asumir la misma comunidad. Asimismo, existe un listado de 800 personas de la zona que necesitan medidas de protección por las amenazas de las que siguen siendo objeto.

Lo anterior rompe con las medidas individuales previstas por las entidades estatales competentes para la salvaguardia de los líderes, insuficientes cuando personas que trabajan en colectivo son amenazadas. Esta incomprensión institucional ha llevado a la coordinación del territorio, conformada por los principales líderes, para desarrollar un plan colectivo para anticipar situaciones de riesgos. Es así como consolidaron una estrategia autónoma de protección humanitaria, en una finca del municipio de Toledo, en donde permanecen líderes que han sido amenazados.

El fin principal de tal coordinación, conforme a las reivindicaciones culturales del Movimiento, es la permanencia de la comunidad en su territorio con dignidad. Si bien las comunidades consideran que deben ser las que piensen la seguridad y la protección para que sea adecuada, requieren del Estado el respaldo de este control territorial y estas medidas de salvaguardia, y ser garante de los que se oponen a este proyecto. Por 
lo tanto, exhortan la toma de medidas diferenciales de carácter políticos, que impliquen el reconocimiento de las particularidades del territorio y generen un ambiente de protección, más allá de las medidas materiales de protección. De esta manera, solamente, se generarían los cambios estructurales requeridos.

En últimas, el movimiento busca posicionar en la opinión pública una reflexión alrededor de la política minero-energética en Colombia y del modelo de desarrollo. Se trata de pensar en otras formas de energía, y de preguntarse ¿para qué se desarrollan estos proyectos?, ¿quiénes se benefician de los mismos?, y ¿a qué costo social y ambiental? Desde la mirada de los líderes, la dificultad a la hora del diálogo con los actores empresariales y estatales reside en un modelo hegemónico de desarrollo en el cual no cabe la diversidad.

\section{NOTAS}

1. https://riosvivoscolombia.org/no-a-hidroituango/lo-que-debes-saber-sobre-hidroituango/, consultado el 10 de febrero de 2021

2. https://www.eltiempo.com/justicia/investigacion/hidroituango-las-razones-por-las-quecontraloria-imputo-cargos-a-fajardo-ramos-y-gaviria-552709, consultado el 10 de febrero de 2021.

3. Sobre el concepto de ecosistemas de violencia, ver intervención de Joséphine Lechartre y Guillermo Trejo, "Mirada comparada: ecosistemas de violencia local en Méjico y Guatemala. Aportes para el caso colombiano" en el Foro "La protección del derecho a la vida de los líderes sociales en Colombia. Una problemática nacional y un desafío internacional", 30 de junio de 2020, consultado el 10 de febrero de 2021.

4. Los estándares interamericanos advierten, entre otros, sobre las consecuencias espirituales y culturales que provocan las afectaciones territoriales por parte de las empresas: https:// www.oas.org/es/cidh/informes/pdfs/EmpresasDDHH.pdf, consultado el 10 de febrero de 2021.

\section{AUTOR}

\section{ISABEL CRISTINA ZULETA}

Isabel Cristina Zuleta es socióloga, ambientalista popular, feminista comunitaria y activista colombiana, vocera del Movimiento Ríos Vivos, que actúa en defensa del territorio y comunidades afectadas por el proyecto hidroeléctrico Hidroituango. En 2018, el Movimiento Ríos Vivos Antioquia recibió el "Premio Defensores" otorgado a organizaciones que dedican su vida a la defensa de sus comunidades originarias. 